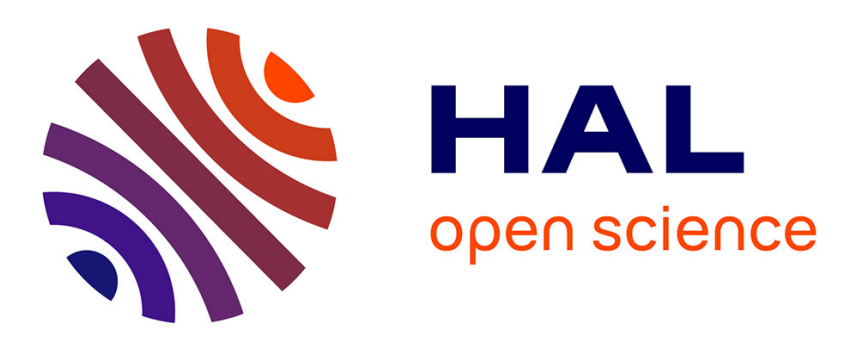

\title{
The pH-Induced Specific Area Changes of Unsaturated Lipids Deposited onto a Bubble Interface
}

Nicolas Anton, Philippe Pierrat, Germain Brou, Gildas Gbassi, Ziad Omran, Luc Lebeau, Thierry Vandamme, Patrick Bouriat

\section{- To cite this version:}

Nicolas Anton, Philippe Pierrat, Germain Brou, Gildas Gbassi, Ziad Omran, et al.. The pH-Induced Specific Area Changes of Unsaturated Lipids Deposited onto a Bubble Interface. Langmuir, 2021, 10.1021/acs.langmuir.0c03046 . hal-03143971

\section{HAL Id: hal-03143971 \\ https://hal.univ-lorraine.fr/hal-03143971}

Submitted on 5 Nov 2021

HAL is a multi-disciplinary open access archive for the deposit and dissemination of scientific research documents, whether they are published or not. The documents may come from teaching and research institutions in France or abroad, or from public or private research centers.
L'archive ouverte pluridisciplinaire HAL, est destinée au dépôt et à la diffusion de documents scientifiques de niveau recherche, publiés ou non, émanant des établissements d'enseignement et de recherche français ou étrangers, des laboratoires publics ou privés. 


\section{The $\mathrm{pH}$-induced specific area changes of unsaturated lipids deposited onto a bubble interface}

Nicolas Anton,,,$++*$ Philippe Pierrat,${ }^{\perp}$ Germain A. Brou, ${ }^{\dagger,}$ Gildas K. Gbassi,, Ziad Omran, ${ }^{\#}$ Luc Lebeau, ${ }^{\dagger}$ Thierry F. Vandamme, ${ }^{\dagger,}$ Patrick Bouriat ${ }^{\S, *}$

${ }^{\dagger}$ Université de Strasbourg, CNRS, CAMB UMR 7199, F-67000 Strasbourg, France

$\$$ INSERM (French National Institute of Health and Medical Research), UMR 1260, Regenerative Nanomedicine (RNM), FMTS, Université de Strasbourg, F-67000 Strasbourg, France

${ }^{\perp}$ Université de Lorraine, CNRS, L2CM UMR 7053, F-57078 Metz, France

『 Université Felix Houphouet Boigny, Chim Phys Lab, 22BP 582, Abidjan 22, Cote Ivoire

\# Department of Pharmaceutical Chemistry, College of Pharmacy, Umm AlQura University, 21955 Makkah, Kingdom of Saudi Arabia

$\S$ CNRS/TOTAL/UNIV PAU \& PAYS ADOUR/E2S UPPA, Laboratoire des Fluides Complexes et leurs Réservoirs -IPRA, UMR5150, 64000 PAU, France.

* To whom correspondence should be addressed: Nicolas Anton (nanton@unistra.fr) and Patrick Bouriat (patrick.bouriat@univ-pau.fr) 


\begin{abstract}
In this work, we used an original experimental setup to examine the behavior of insoluble monolayers made with $\mathrm{pH}$-sensitive lipids. Two kinds of unsaturated lipids were chosen: a cationic one (lipid 1) bearing an ammonium head group and an anionic one (lipid 2) terminated with an acidic phenol group. The lipids were deposited onto an air bubble interface maintained in an aqueous phase and, after stabilization, were subjected to a series of compressions performed at different $\mathrm{pH}$ values. These experiments disclosed a gradual increase in the specific area per molecule when lipids were neutralized. Imposing a $\mathrm{pH}$ variation at constant bubble volume also provided surface pressure profiles that confirmed this molecular behavior. As complementary characterization, dilatational rheology disclosed a phase transition from a purely elastic monophasic system to a viscoelastic two-phase system. We hypothesized that this unexpected increase in the specific area with lipid neutralization is related to the presence of unsaturations in each of the two branches of the hydrophobic tails that induce disorder, thereby increasing the molecular area at the interface. Application of the two-dimensional Volmer equation of state allowed the generation of quantitative values for the specific areas that showed variations with $\mathrm{pH}$. It also allowed the determination of apparent $\mathrm{pK}_{\mathrm{a}}$ values, which are affected by both the electrostatic potential within the monolayer and the affinity of the lipid polar head for the aqueous phase.
\end{abstract}




\section{Introduction}

The clinical use of chemotherapeutics in the treatment of cancer is effective, but it is also associated with drawbacks in terms of the benefit-to-risk balance. The two main limitations of these drugs are their lack of specificity for the target tissue and the multidrug resistance (MDR) of many tumors. ${ }^{1}$ Designing drug carriers that specifically deliver a drug to the tumor site is of particular interest, as this would significantly improve the drug's selectivity and reduce the severe side-effects associated with chemotherapy. One important characteristic of the microenvironment of solid tumors is the modification of the $\mathrm{pH}$, i.e., the $\mathrm{pH}$ decreases in the extracellular medium and increases in the cytosol. ${ }^{2-4}$ Thus, exploiting the $\mathrm{pH}$ near tumors to trigger drug release is a promising targeting strategy. ${ }^{5}$ At this point, understanding the behavior of $\mathrm{pH}$-sensitive lipids is crucial to optimize the efficiency of these drug delivery systems.

The most representative example of this drug delivery strategy is the use of liposomes made with lipids containing the phosphatidylethanolamine (PE) polar head, as this structure imparts $\mathrm{pH}$ sensitivity and their bilayer rupture under acidic conditions due to a phase transition of the membrane. ${ }^{6-11}$ Pure PE-lipid bilayers, however, are not stable and need a stabilizer like alphatocopheryl succinate ( $\alpha$-TOS), oleic acid, palmitoylhomocysteine, or cholesteryl hemisuccinate (CHEMS). ${ }^{10,12-14}$ Previous reports have emphasized the impact of the sub-phase $\mathrm{pH}$ on the bilayer phase transition, as this $\mathrm{pH}$ results in membrane destabilization and liposome destruction. We recently reported a comprehensive study investigating the stability of liposomes in response to $\mathrm{pH}$ variations and the influence of liposome composition on this behavior. Manipulation of the chemical nature and concentration of PE-lipids and stabilizers allows a fine equilibration between membrane stability and $\mathrm{pH}$ sensitivity, thereby allowing the tailoring of liposome properties and enabling controlled drug release. ${ }^{11}$

Such a study of liposome integrity to investigate the behavior of $\mathrm{pH}$-sensitive lipids is an indirect approach. By contrast, the present work proposes a complementary approach based on the direct investigation of the thermodynamic behavior of monolayers made with $\mathrm{pH}$-sensitive lipids, with the aim of understanding the relationship between $\mathrm{pH}$ and lipid phase transition. While the study of insoluble monolayers is generally set up in Langmuir troughs, we propose its transposition to a classical axisymmetric drop shape analysis (ADSA) technique. Our previously developed methodology ${ }^{15}$ allowed the study of insoluble monolayers deposited on a bubble interface and has two advantages: (i) the composition of the bulk phase can be controlled very easily through a bulk phase exchange in the cuvette, and (ii) the method requires only a very small amount of lipid for each experiment. In addition, this experimental configuration allows to perform successive isothermal compressions of the same monolayer, with a gradual modification of the bulk composition $(\mathrm{pH})$, through a very simple protocol.

The lipids used in our investigations display a $\mathrm{pH}$-sensitive (i.e., titratable) polar head (Fig. 1). ${ }^{16}$ Compound $\mathbf{1}$ exists as a cationic species at acidic $\mathrm{pH}$ (ammonium form), whereas compound $\mathbf{2}$ exists as an anionic one at basic $\mathrm{pH}$ (phenate form); lipid $\mathbf{2}$ incorporate three ethylene oxide units that reinforce the amphiphilic properties. These lipids were deposited onto the water/air interface of a bubble in the ADSA experimental setup and, after washing and stabilization (see full details in the Materials and Methods section), the bubble interfacial area was gradually reduced and the monolayer compressed. The surface pressure was monitored and the compression isotherm established. A further set of experiments was conducted at constant bubble interfacial area, but with gradual changes in the bulk phase $\mathrm{pH}$, to measure the surface pressure and Gibbs monolayer elasticity. These complementary approaches clearly revealed the 
phase transitions and monolayer behaviors as a function of initial compression (i.e., monolayer density), as well as the potential change in the molecular organization at the interface induced by $\mathrm{pH}$ modification. By choosing lipids differing by their polar head, the idea was to compare different $\mathrm{pH}$-sensitive lipids, mainly cationic and anionic, but also with difference in their structures, and eventually draw parallel between results, in order to find similitudes in their interfacial behavior.



Figure 1: Structures of the two titratable custom-made model lipids investigated in this study. Lipid 1 is 3(dimethylamino)propane-1,2-diyldioleate, and lipid $\mathbf{2}$ is 2,3-bis(oleoyloxy)propyl(2-(2-(2-((3-chloro-4hydroxybenzoyl)oxy)ethoxy)ethoxy)ethyl) succinate.

The experimental approach developed herein can be regarded as a new way to study insoluble monolayers and characterize their complex behavior at the air-water interface, as a function of the composition of the subphase, and as well as along a gradual modification of this composition.

\section{Materials and methods}

\section{Chemicals}

Unless otherwise stated, all chemical reagents were purchased from Alfa Aesar (Bischeim, France) and were used as received. All buffer solutions were prepared with deionized water purified with an EMD Millipore Milli-Q integral system (resistivity $\leq 18.2 \mathrm{M} \Omega \cdot \mathrm{cm}^{-1}$ ) and filtered through a $0.2 \mu \mathrm{m}$ polycarbonate membrane. The synthesis and study of the respective ionization behavior of cationic lipid 1 and anionic lipid 2 in liposomes by fluorescence spectroscopy has been recently reported. ${ }^{16}$ The synthesis of these lipids was performed starting from oleic acids, i.e. cis-9-octadecenoic acid (Sigma Aldrich, purity $\geq 99 \%$ ). Noteworthy, neither in the synthesis process, nor in the characterization methods described below, operating conditions could allow cis to trans isomerization of the double bonds. Furthermore, the lipids were characterized by ${ }^{1} \mathrm{H}$ NMR and definitely confirmed the absence of trans isomerics. ${ }^{16}$

\section{Surface tension measurements}


The axisymmetric drop shape analysis method (ADSA), using a Tracker drop tensiometer (Teclis, Longessaigne, France), was used to measure the dynamic surface tension $\gamma$ at the air/water interface. The ADSA is an absolute method, so the surface pressure $\Pi$ is obtained simply as the difference between the free water-air surface $\left(\gamma_{0}\right)$ and the covered surface $(\gamma)$. A rising bubble (air) was formed in ultrapure water (MilliQ Filtration System), with both volume and temperature control. The bubble shape was recorded in real time with a video camera, and its Laplacian shape gave the values of interfacial area and surface tension. All the experiments were performed in triplicate at $20^{\circ} \mathrm{C}$.

\section{Lipid deposition and monolayer formation}

The study of insoluble monolayers deposited onto the interface of the rising bubble (illustrated in Fig. 2) in a drop tensiometer is an original method we recently developed. ${ }^{15}$ Compared to conventional techniques used to study insoluble monolayers (e.g., the Langmuir trough method), this approach presents two very important advantages: (i) the easy experimental configuration for performing interfacial rheology and accessing the viscoelastic properties of the monolayer, and (ii) the facile modification of the composition of the aqueous phase during measurement, which is a fundamental point in the present study as it allows very fine tuning in the bulk phase $\mathrm{pH}$ without replacement of the monolayer.

The cuvette was equipped with a water-renewing system using two peristaltic pumps for circulation (flow rate: $22 \mathrm{~mL} / \mathrm{min}$ ) (see Fig. 2). The insoluble amphiphiles were solubilized beforehand in chloroform at $193 \mu \mathrm{M}$ and withdrawn into a micro-syringe $(10 \mu \mathrm{L})$. A very small volume $(<0.25 \mu \mathrm{L})$ of this chloroform solution containing the lipid was deposited onto the air bubble previously formed and which volume is maintained at $6 \mu \mathrm{L}$. The organic phase spread at the bubble surface, and the needle used to deposit the organic phase was rapidly removed from the solution. The bubble volume was then mechanically controlled and set at $8 \mu \mathrm{L}$. Bulk phase water flow (which was turned off during the deposition step) was then turned on to extract and remove chloroform. At this stage, the water circuit was opened (not like in Fig. 2), and the extracted water was flushed out and replaced with fresh water. After 10 minutes, the bubble volume was gradually set to $13 \mu \mathrm{L}$, corresponding to the gaseous state of the lipid monolayer and $\gamma$ stabilized at $72 \mathrm{mN} / \mathrm{m}$. Water flow was maintained for $1000 \mathrm{~s}$ to stabilize the monolayer, and then the water circulation stopped, as shown in Fig. 2. The cuvette was connected to an external beaker that used to add small amounts of $\mathrm{HCl}(0.1 \mathrm{M})$ or $\mathrm{NaOH}(0.1 \mathrm{M})$ to modify the $\mathrm{pH}$ of the whole circuit. The external beaker could also contain the electrode of a $\mathrm{pH}$ meter (as depicted in the figure). 


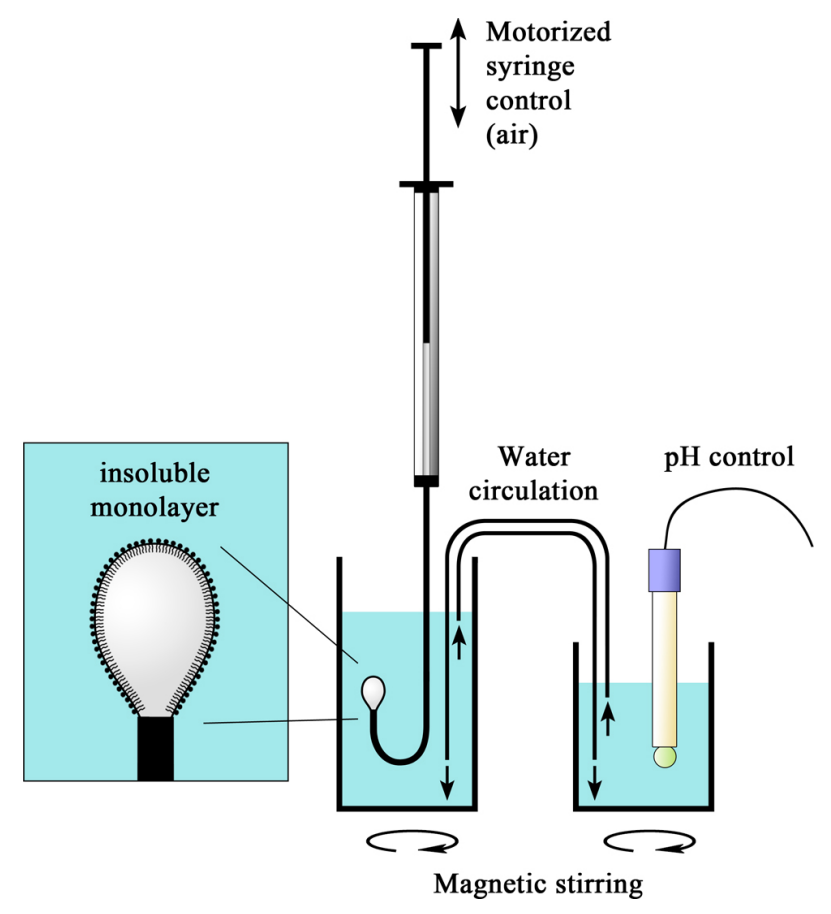

Figure 2: Experimental setup for the study of insoluble monolayers, compression experiments, and interfacial rheology. Bubble is setup in the cuvette and the water circulation allows modifying the bulk phase composition and real-time $\mathrm{pH}$ measurement.

\section{Compression experiments}

After the monolayer was formed (vide supra), the $\mathrm{pH}$ was brought to values corresponding to the ionized lipid form. In the case of cationic lipid $1,1 \mathrm{~mL}$ of $\mathrm{HCl}(0.1 \mathrm{M})$ was added to the external beaker to give a $\mathrm{pH}$ around 3.1, while in the case of anionic lipid 2, $0.7 \mathrm{~mL}$ of $\mathrm{NaOH}$ $(0.1 \mathrm{M})$ was added to increase the $\mathrm{pH}$ to around 10.1 . The water circulation was maintained for $3600 \mathrm{~s}$ to ensure a homogeneous composition of the bulk phase, as well as the stabilization of the monolayer in its ionized form. Then it was stopped and the monolayer compression performed by decreasing the bubble volume from $13 \mu \mathrm{L}$, to either $2-3 \mu \mathrm{L}$ or to reaching a tension of $25 \mathrm{mN} / \mathrm{m}$. The compression process consisted of a linear decrease in the bubble volume over time, according to a compression rate of approximately $3 \mathrm{~mm}^{2} / \mathrm{min}$. As described in our previous study, ${ }^{15}$ during monolayer compression, the Bond number $(\mathrm{Bo})$ retained a suitable value $B o>0.15$, confirming the applicability of the algorithm used in the ADSA method. When the compression was complete, the bubble volume was slowly brought back to $13 \mu \mathrm{L}$. Control experiments were punctually performed at constant $\mathrm{pH}$, showing the superimposition of the curves, and proving that compressions did not induce expulsion of lipid molecules in the bulk phase, i.e. decrease of interfacial concentration. Regarding the stability of lipids, $\mathrm{pH} 3$ and 10 could be considered as diluted acidic $\left(10^{-3} \mathrm{~mol} / \mathrm{L} \mathrm{HCl}\right)$ or basic $\left(10^{-4}\right.$ $\mathrm{mol} / \mathrm{L} \mathrm{NaOH})$ aqueous solution, which are not expected to promote ester hydrolysis on a chemical point of view, especially at room temperature (as shown in previous works ${ }^{16}$ ).

The presence of an electrolyte in the solution, e.g. $\mathrm{NaCl}(50 \mathrm{mM})$, was necessary for the use of a $\mathrm{pH}$ electrode. However, since we observed that the presence of salt in the bulk phase induced 
a slight shift in the compression curves, the first compression with fully ionized amphiphiles was performed in pure water and served as reference for further analyses. Therefore, $2 \mathrm{~mL} \mathrm{NaCl}$ $(2.8 \mathrm{M})$ was added to the external beaker to set the electrolyte concentration at $50 \mathrm{mM}$ and allowed the measurement and monitoring of the $\mathrm{pH}$ during throughout the experiment. The water circulation was turned on and the monolayer was allowed to stabilize for $1800 \mathrm{~s}$ before stopping the circulation and performing a second compression. The same protocol was followed, after addition of a new volume (variable in function of the $\mathrm{pH}$ ) of $\mathrm{NaOH}(0.1 \mathrm{M})$ or $\mathrm{HCl}(0.1 \mathrm{M})$ to the external beaker, for cationic or anionic lipid, respectively. The results were therefore a set of different compression curves at different $\mathrm{pH}$ values going from the ionized form of the lipid toward its neutral configuration. It is important to note that in such drop tensiometer experiments, a recurrent difficulty lies in the exact reproducibility of the experimental conditions. Herein, it is precisely the case, and for several reasons: first, the amount of deposited material cannot be precisely controlled, thus the isothermal compressions can be shifted from one experiment to the other (on the x-axis); second, the experimental points of the surface tension as a function of time and volume are not strictly recorded at the same time and same drop volume, between two different experiments; and third, upon addition of $\mathrm{NaOH}$ (or $\mathrm{HCl}$ ), the values of $\mathrm{pH}$ are also not strictly similar between two experiments, since the medium is not buffered. This is the main reason why the experimental results reported herein are representative of the behavior observed in experiments that were always repeated at least three times.

\section{Interfacial rheology}

Sinusoidal variations of the bubble volume were imposed through the syringe piston, and the sinusoidal surface tension response was recorded, providing the dilatational elastic modulus. The selected frequency was $0.0166 \mathrm{~Hz}$ and the amplitude of the imposed sinusoidal volume variation did not exceed $10 \%$ of the bubble volume. The sinusoidal solicitations were performed with small amplitudes to ensure that all measurements were in the linear viscoelastic regime.

In contrast with the compression experiments described above, and in order to study the direct impact of the $\mathrm{pH}$ change on the monolayer properties, interfacial rheology measurements were carried out at a fixed compression at different $\mathrm{pH}$ values. The cationic lipid (lipid 1) was deposited following a similar protocol to that described above: (i) after lipid deposition at the bubble surface, the monolayer was washed for $1000 \mathrm{~s}$, (ii) the water circulation circuit was closed and turned on, (iii) the $\mathrm{pH}$ was decreased to about $\mathrm{pH} 3.1$ (by adding $1 \mathrm{~mL} 0.1 \mathrm{M} \mathrm{HCl}$ to the external beaker), (iv) the monolayer was allowed to stabilize for $3600 \mathrm{~s}$ under water circulation, $(v)$ the compression was performed up to a selected value of $\Pi_{0}=5,7,9,20$ or $30 \mathrm{mN} / \mathrm{m}$, (vi) the $\mathrm{NaCl}$ concentration was set at $50 \mathrm{mM}$ and the monolayer was stabilized for $1800 \mathrm{~s}$, (vii) the value of the surface tension was recorded and rheology experiments were performed at $f=0.016 \mathrm{~Hz}$ to obtain the elastic modulus $E$ and the loss angle $\phi$ (angle between E' and E" in the complex plane). The theoretical aspects regarding their evaluation from experimental data with a drop tensiometer are detailed in previous work, ${ }^{17}$ (viii) a volume of $0.1 \mathrm{M} \mathrm{NaOH}$ was added to induce a $\mathrm{pH}$ increase of the bulk phase, and the monolayer was allowed to stabilize for $1800 \mathrm{~s}$. Steps (vii) and (viii) were repeated to cover the desired $\mathrm{pH}$ range. The whole protocol was repeated three times for a given value of $\Pi_{0}$, and the whole procedure was repeated again with other values of $\Pi_{0}$. 


\section{Results and discussion}

\section{Impact of the bulk phase $\mathrm{pH}$ on the lipid monolayers at the air/water interface}

Let us first focus on the study of a monolayer formed with the cationic lipid $\mathbf{1}$ deposited at the air/water interface of the rising bubble of the tensiometer. The results are summarized in Fig. 3, which shows the compression experiment (Fig. 3a) and the corresponding values of surface pressure performed at constant bubble volume (Fig. 3b). The initial compression at $\mathrm{pH}=3.16$ in ultrapure water shows a concave curve regularly growing along with compression. At this low $\mathrm{pH}$, the lipid head groups are largely ionized and the monolayer should behave as a liquid expanded (LE) homogeneous phase (curve (o)). Upon addition of electrolyte $(50 \mathrm{mM} \mathrm{NaCl}$ in bulk phase), the curve retains the same profile but is shifted to a higher bubble area (phase $(i)$ ). The same concave shape indicates that, in presence of salt, the monolayer is still homogeneous. An additional shift is observed when the $\mathrm{pH}$ is increased to 3.85 and 4.63.

At higher $\mathrm{pH}$ values (starting at $\mathrm{pH}=6.23$, phase (ii)), the curves exhibit a clear break (a shoulder) at $\Pi=27 \mathrm{mN} / \mathrm{m}$. This transition is typical of a phase transition. The fact that the lower part of the curve is still shifting to the right along with $\mathrm{pH}$ (as mentioned above) indicates that, at low surface pressures, the monolayer is still a homogeneous mixture of neutral and ionized lipids, but contains increasingly more neutral molecules as the $\mathrm{pH}$ increases.

The fact that the plateau is not horizontal can be caused either by kinetic effects, as continuous compression does not allow the system to strictly reach equilibrium, or by impurities, the neutralized lipids may play this role, forming a second phase. Thus, we assume that the shoulder reveals the formation of a second phase, which presumably is formed by clusters of segregated neutral lipids (favored by the $\mathrm{pH}$ increase); these neutral lipids, above a given surface pressure threshold $(\sim 27 \mathrm{mN} / \mathrm{m})$, gather and induce a phase separation. The fact that the phase change does not occur at a constant surface pressure (absence of a plateau) does support that the new phase may be a heterogeneous mixture of segregating neutral and ionized lipids. ${ }^{18}$ 

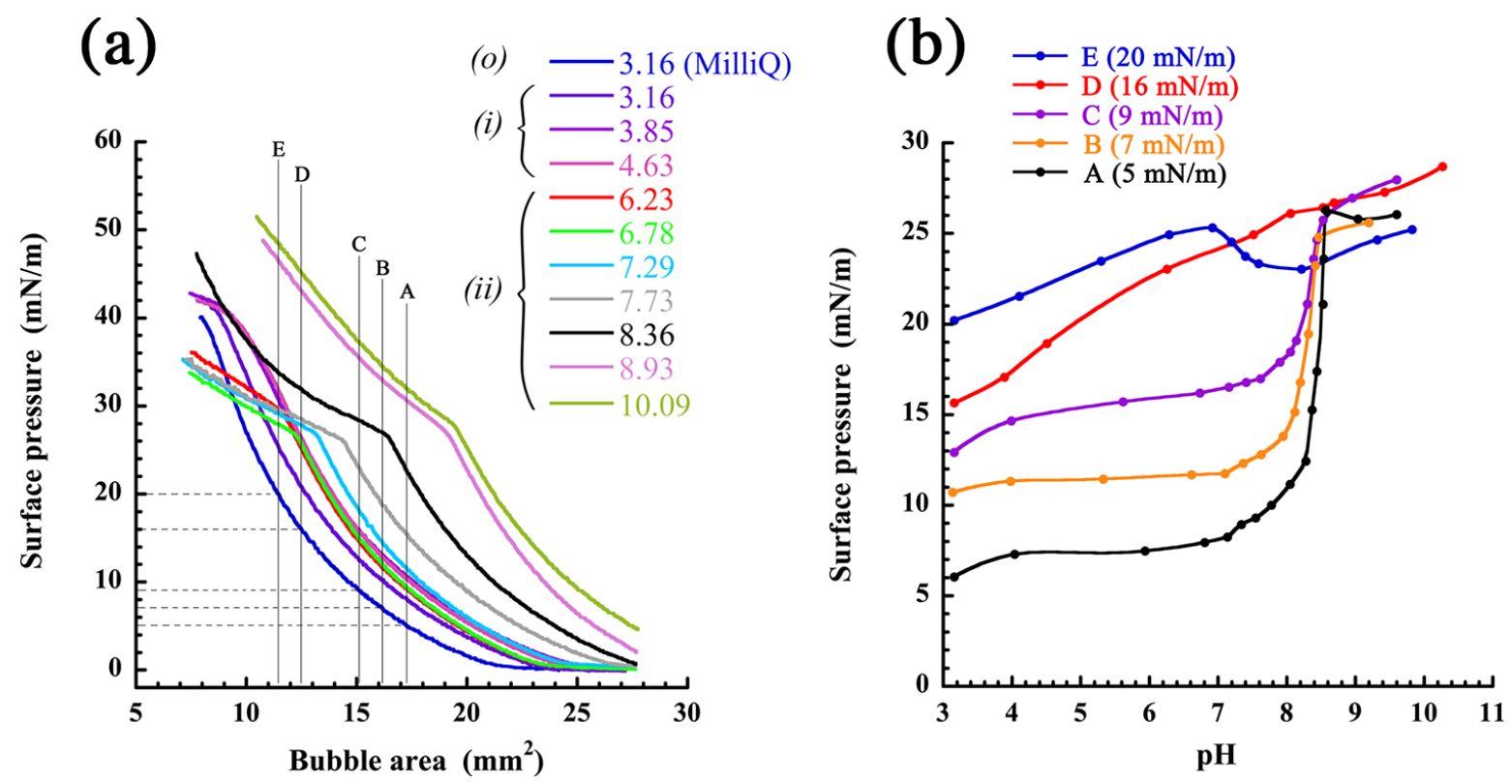

Figure 3: Study of cationic lipid 1 at the air/water interface, as a function of $\mathrm{pH}([\mathrm{NaCl}]=50 \mathrm{mM})$. (a) For compression experiments, each monolayer compression was performed at constant $\mathrm{pH}$. The indication "MilliQ" means the bulk phase is pure MilliQ water before addition of $\mathrm{NaCl}$. (b) Values of surface pressure as a function of $\mathrm{pH}$. Each curve is generated at constant bubble volume, fixed with the initial monolayer compression noted in the figure legend (i.e. $\Pi_{0}=5,7,9,16$ and $20 \mathrm{mN} / \mathrm{m}$ ). The different compression states presented in figure (b) are shown in figure (a) as vertical lines, showing the evolution of the monolayer when only the $\mathrm{pH}$ is changed.

The main result disclosed in Fig. 3 is the increase in the specific molecular area upon addition of $\mathrm{NaCl}$ and the $\mathrm{pH}$ increase. Interestingly, this behavior has not been reported in the literature for monovalent phospholipids. ${ }^{19}$

These curve shifts result from $\mathrm{NaCl}$ or $\mathrm{pH}$ increases. One hypothesis would be that this shift is related to the change in the intermolecular electrostatic repulsion, expressed as an electrostatic contribution in the surface pressure. However, Levental et al. ${ }^{19}$ have shown that this electrostatic contribution is negligible in monolayers made with phospholipids having a valence lower than 2. In that case, when the distance between the two closest molecules is higher than the Bjerrum length $\left(l_{B} \sim 7 \AA\right)$, the energy of electrostatic repulsion is lower than the thermal agitation ( $k_{B} T$, where $k_{B}$ is the Boltzmann's constant and $T$ the temperature). ${ }^{19}$ It follows that the electrostatic contribution of the surface pressure is negligible and therefore compatible with the application of the Volmer equation of state, as proposed below. This is true below the plateau when the electrostatic contribution of the surface pressure is effectively neglected when the overlapping of molecules is low. However, above the plateau, the monolayer is compressed and the electrostatic compression occurs along with the phase separation.

In addition, another experimental observation point corroborates this hypothesis: if the electrostatic interactions play a role in the compression curve, the surface pressure should decrease with the $\mathrm{pH}$ (along with the gradual neutralization of lipids). ${ }^{19,20}$ However, an opposite effect is observed, meaning that another phenomenon predominates. Therefore, we will assume in the following discussion that the electrostatic surface pressure is negligible and that the shifts 
in the compression isotherms come from an indirect mechanism, possibly related to conformational changes. Interestingly, both behaviors are reported in literature: on the one hand, as expected, electrostatic repulsion at interface induces an increase of the area per molecules, ${ }^{19}$ as observed for instance with calixarenes. ${ }^{20}$ On the other hand, however, other reports showed that a neutralization of the molecular charges (studying dioleoylphosphatidylcholine) induces a monolayer expansion, in agreement with our results. ${ }^{21,22}$

In order to confirm this observation (and our experimental procedure), a second set of experiments was conducted at a constant bubble volume while varying $\mathrm{pH}$. The results are reported in Fig. 3b. The initial monolayer compression was performed at $\mathrm{pH}=3.16$ without $\mathrm{NaCl}$, and stopped at chosen pressure values of $\Pi_{0}=5,7,9,16$, or $20 \mathrm{mN} / \mathrm{m}$ (curves A, B, C, $\mathrm{D}$, or $\mathrm{E}$, respectively). These values of $\Pi_{0}$ can be read on the ordinate of horizontal dashed lines in Fig. 3a respective to the initial compression curve in blue for $\mathrm{pH}=3.16$ without $\mathrm{NaCl}$. $\mathrm{NaCl}$ $(50 \mathrm{mM})$ was added to follow the $\mathrm{pH}$ variations.

Therefore, the crosses between vertical gray lines and isotherms reported in Fig. 3a correspond to the change of $\Pi$ in function of $\mathrm{pH}$ at constant bubble area, and should actually correspond to the results given in Fig. $3 b$. The values of $\Pi$ disclosed in Fig. 3 (b) follow the trend disclosed by these vertical lines in Fig. 3a. For curves A, B, C, and D, the values of $\Pi$ show a constant increase, while the shape of curve $\mathrm{E}$ shows a maximum due to the particular location of the vertical line that crosses compression curves that intersect with each other. These two complementary experiments show similar trends, thereby confirming the behavior of the compression curves made at different $\mathrm{pH}$ presented in Fig. 3a. Note that the values of $\Pi$ noticeably differ between Fig. 3a and $3 \mathrm{~b}$ for high surface pressures and $\mathrm{pH}$ (e.g. curve E in Fig. $3 \mathrm{~b})$. This is simply due to the fact that the values of the pressure in the compression curves in Fig. 3a are instantaneous values, while in Fig. 3b, they are measured at equilibrium, after $1800 \mathrm{~s}$ of monolayer stabilization. This can also explain why, at equilibrium, the surface pressure never exceeds $30 \mathrm{mN} / \mathrm{m}$, even at high $\mathrm{pH}$ (as opposed to what we can see on compression curves). In addition, the good agreement between the compression experiments (Fig. 3a) and the $\mathrm{pH}$ variation studies (Fig. 3b) confirms the increase in the specific area of lipid $\mathbf{1}$ upon neutralization due to the $\mathrm{pH}$ increase.

A modification of the molecule orientation at the interface is an explanation compatible with the observed interfacial expansion. At low $\mathrm{pH}$, the ionized lipids (lipid 1, or DOPC in previously reported studies ${ }^{21,22}$ ) are pulled toward the aqueous bulk phase due to the solvation of their charged polar heads. Their two hydrophobic tails are then forced to get closer to minimize the solvation energy by water, thereby decreasing the apparent value of the mean specific area per molecule $\omega$. Conversely, the neutral lipids at high $\mathrm{pH}$ lose affinity for the bulk phase, i.e. they are more confined at the water/air interface. The aliphatic chains can now deploy under the effect of thermal agitation, occupying an even higher place by virtue of their twisted shape due to the presence of unsaturation (for lipid 1 and DOPC). The value of the apparent $\omega$ is eventually increased compared to the ionized state. This behavior is schematically represented and summarized in Fig. 4, which shows the ionized lipids as small red disks and neutral lipid as larger blue ones, and for homogeneous and bi-phasic states (Fig. $4 \mathrm{a}$ and 4b, resp.). The representation describing the lipid parts as flat lying in water is admitted in literature, where, at a water/air interface, the trend is to expulse the aliphatic chain towards the interface. ${ }^{23}$ 
(a)

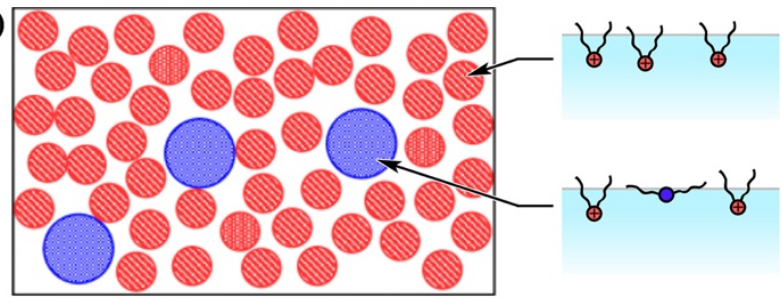

(b)

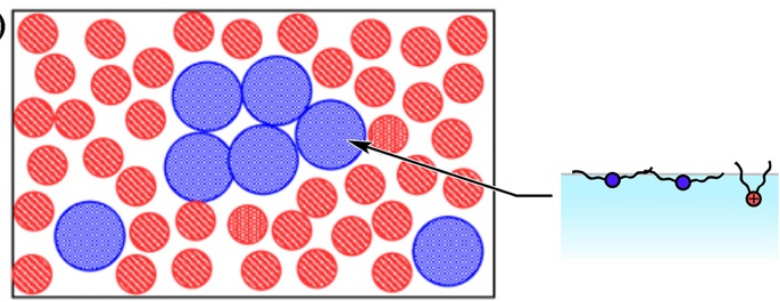

Figure 4: Schematic representation of the interfacial organization of the cationic lipids as a function of $\mathrm{pH}$ range and compression region. Red and blue disks represent ionized and neutral molecules at the interface, respectively. Configuration (a) corresponds to the homogeneous state $(\mathrm{pH}<6$ or before the phase transition for higher $\mathrm{pH}$ ): a homogeneous mixture of ionized and neutral lipids; and configuration (b) corresponds to the bi-phasic state after the phase transition $(\mathrm{pH}>6$ and $\Pi>27 \mathrm{mN} / \mathrm{m})$ : neutral molecules gathering as clusters, coexisting with a homogeneous mixture of ionized and neutral lipids.

This schematic representation illustrates the differences in the values of $\omega$ between the ionized and neutral forms of lipids. It could explain the continuous shift of the curves as the $\mathrm{pH}$ is increased, as it is linked to the increase in the average $\omega$, along with the conversion of ionized to neutral configurations. It follows that the global shift of the curve highlights the gradual transition between the ionized and neutral states of the lipids and eventually can help to determine the values of an apparent interfacial $\mathrm{pK}_{\mathrm{a}}$, the so-called $\mathrm{pK}_{\mathrm{a}}^{\mathrm{app}}$, associated with this ionization. Accordingly, this $\mathrm{pK}_{\mathrm{a}}^{\mathrm{app}}$. value can be evaluated by expressing the bubble area that gives a constant given surface pressure within the homogeneous domain (i.e., below $27 \mathrm{mN} / \mathrm{m}$ ). This representation for cationic lipid 1 studied above in Fig. 3, is reported in Fig. 5. In addition, in order to show that this behavior does not depend on $\Pi$, the curves were established for $\Pi=$ 5,10 and $20 \mathrm{mN} / \mathrm{m}$ (still in the homogeneous phase). A typical sigmoidal curve shape is observed, showing a same $\mathrm{pH}$ transition whatever the surface pressure $\Pi=5,10$ or $20 \mathrm{mN} / \mathrm{m}$, meaning that the ionized/neutral transition is independent of the surface charge density. This reinforces the assumption for which the electrostatic contribution of the surface pressure is negligible at low surface pressure. Then, the $\mathrm{pK}_{\mathrm{a}}^{\mathrm{app}}$. should approach the value of $\mathrm{pK}_{\mathrm{a}}$ in solution, at around 8.5 in Fig. 5 (see arrow). This value is close to the one expected for the amino function and also to the apparent $\mathrm{pK}_{\mathrm{a}}$ of lipid $\mathbf{1}$, as determined by a fluorescence method in similar conditions in our previous study ${ }^{16}$ that gave experimentally a value of 7.01 and a predicted value of 8.02 (using the $\mathrm{ACD} / \mathrm{pKa}$ v 6.00 software, $\mathrm{ACD} /$ Laboratories, Toronto, $\mathrm{ON}$, Canada). 


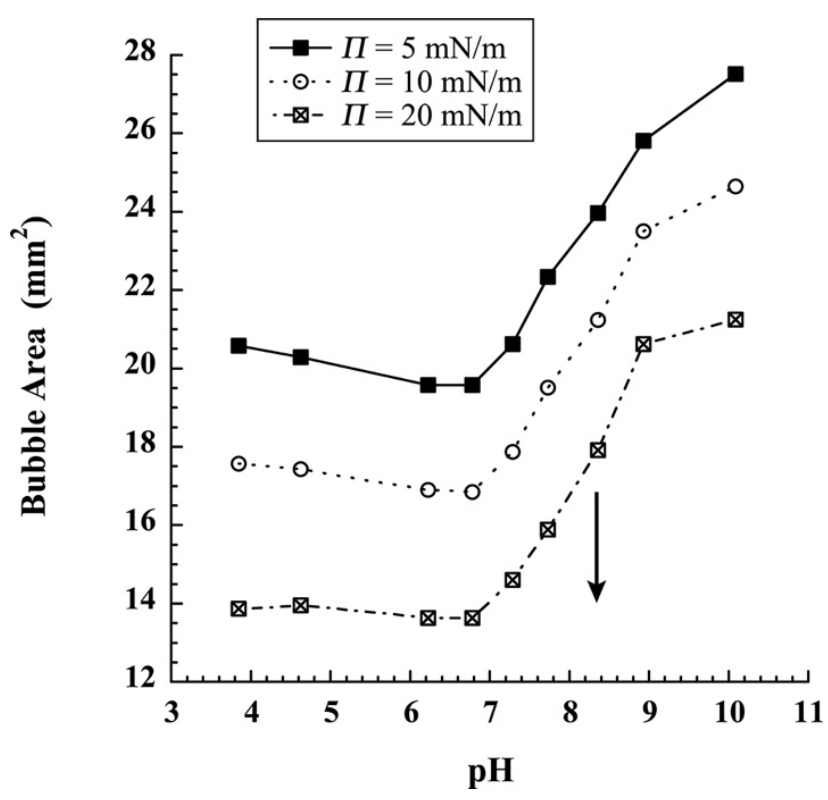

Figure 5: Analysis of the curve shift form compression curves Fig. 3 (a) for cationic lipid 1. The bubble area is expressed at a constant pressure (following horizontal lines) as a function of $\mathrm{pH}$.

\section{Dilatational properties of the lipid monolayers as a function of the bulk phase pH}

The results and their interpretation presented in the previous section are mainly based on the compression curves and the study of their evolution with $\mathrm{pH}$. Rheological study of the lipid monolayer was therefore performed to confirm these results. The dilatational properties of the monolayer of cationic lipid 1 were determined under similar experimental conditions to those chosen for the surface pressure experiments in Fig. $3 \mathrm{~b}$, for values of the initial compression $\Pi_{0}$ $=5,7,9,16$ or $20 \mathrm{mN} / \mathrm{m}$. The results reported in Fig. 6 show the elastic modulus $E$ in Fig. $6 \mathrm{a}$ and the corresponding loss angle $\phi$ in Fig. $6 \mathrm{~b}$. 

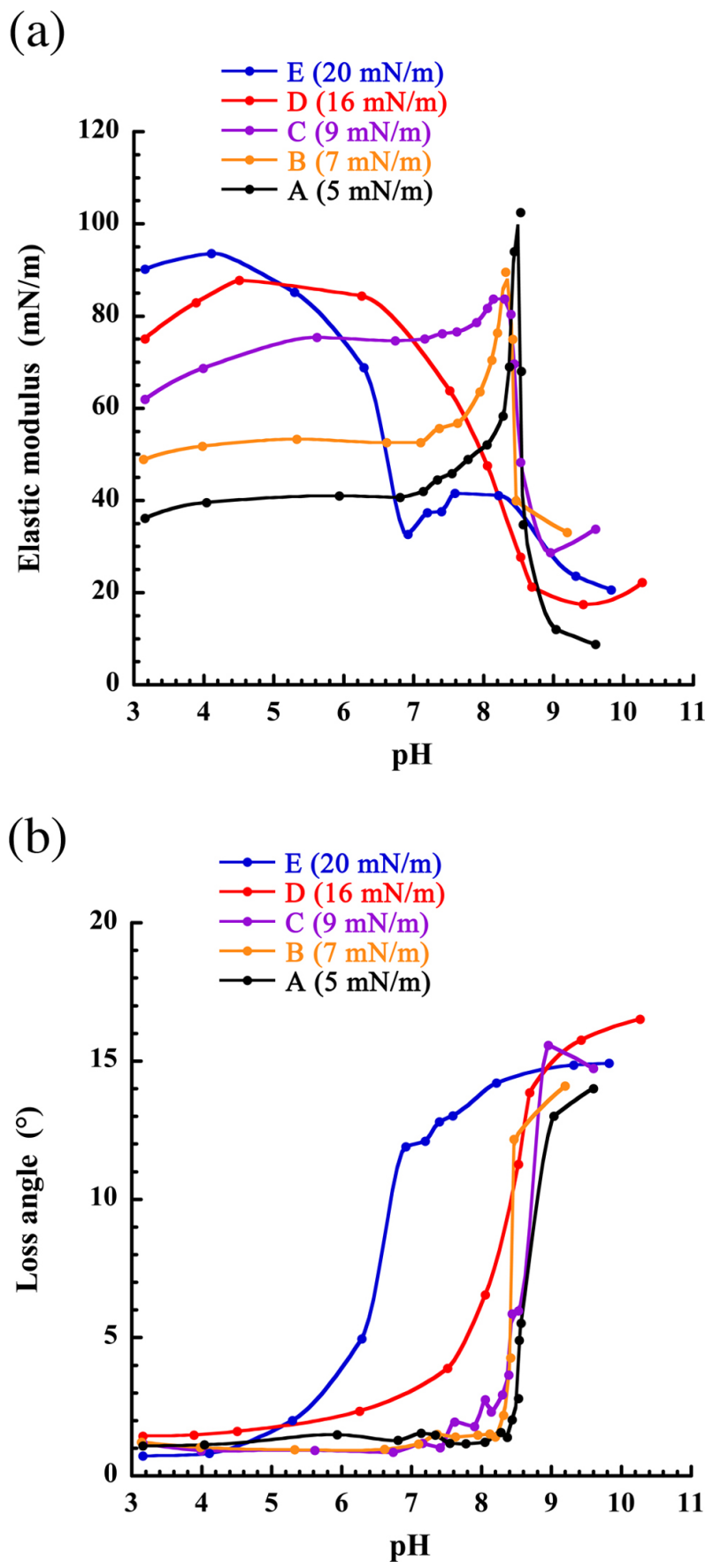

Figure 6: Dilatational rheology experiments of monolayers of cationic lipid $\mathbf{1}$ at the air/water interface as a function of $\mathrm{pH}([\mathrm{NaCl}]=50 \mathrm{mM})$. (a) Elastic modulus as a function of $\mathrm{pH}$. Each curve is generated at a constant bubble volume, fixed with the initial monolayer compression noted in the figure legend (i.e. $\Pi_{0}=5,7,9,20$ and $30 \mathrm{mN} / \mathrm{m}$ ). (b) Corresponding values of the loss angle as a function of $\mathrm{pH}$.

For experiments conducted at the lowest surface pressure (Fig. 6, curves A, B and C), the value of $E$ gradually increases to form a very narrow peak located at $\mathrm{pH}=8.5$, coinciding with the $\mathrm{pK}_{\mathrm{a}}$ value determined above. Actually, the existence of a maximum value for elastic modulus as a function of $\mathrm{pH}$ is expected, regarding the compression curves in Fig. 3a. In the absence of relaxation, the instantaneous elastic modulus $E_{\infty}$ is defined by Gibbs in Eq. (1), where $A_{\mathrm{B}}$ is the bubble area with a constant number of molecules at interface $N$ and constant temperature $T$ : 
$E_{\infty}=-A_{B}\left(\frac{\partial \Pi}{\partial A_{B}}\right)_{T, N}$

Therefore, the instantaneous elastic modulus is directly linked to the slope of the compression curves $\Pi-A_{\mathrm{B}}$ shown in Fig. 3a. As the $\mathrm{pH}$ is increased (i.e., when moving along the vertical lines in Fig. 3a), the values of elastic modulus can be read as the successive slopes of compression curves at the intersections of vertical lines and compression curves. This held true as long as the loss angle remained weak (i.e., in absence of dissipative process in the monolayer). For curves A, B, and C, the slopes gradually increase while the monolayers are in the homogeneous phase, to reach a maximum (corresponding to the peak of elasticity) just before the shoulders, and then fall when entering the two-phase region. The left part of the peaks, in Fig. 6a, therefore appears to correspond to the homogeneous phase, while the right part corresponds to the two-phase region. In addition, the values of elastic modulus between 30 and $100 \mathrm{mN} / \mathrm{m}$, confirmed the nature liquid-expanded phase state. ${ }^{24}$

In curves $\mathrm{A}, \mathrm{B}$, and $\mathrm{C}$, the loss angle suddenly changes from zero to a value of around $15^{\circ}$ when $\mathrm{pH}>\mathrm{pK}_{\mathrm{a}}$. Before $\mathrm{pK}_{\mathrm{a}}\left(\right.$ when $\phi \sim 0^{\circ}$ ), the homogeneous phase is purely elastic, as should be the case for a gaseous state made of insoluble molecules. Beyond $\mathrm{pK}_{\mathrm{a}}\left(\phi \sim 15^{\circ}\right)$, the significant increase of $\phi$ shows that the two-phase monolayer is able to relax. In general, the non-negligible values of the loss angle mean that the amphiphiles undergo adsorption/desorption phenomena. However, as these lipids (whether they are ionized or not) are not soluble, this is not the case here. Accordingly, the relaxation mechanism should be related to a two-dimensional reorganization of the lipids. These dilatational rheology results confirmed the homogeneous/two-phase transition observed in the isotherms: as the $\mathrm{pH}$ increases, the number of neutralized lipids also increases and eventually triggers the lipid segregation in the monolayer. This relationship between loss angle and phase transition was also analyzed in literature reports, ${ }^{25}$ corroborating our explanation.

By contrast, at a higher initial compression $\left(\Pi_{0}=16\right.$ and $20 \mathrm{mN} / \mathrm{m}$, curves $\mathrm{D}$ and $\left.\mathrm{E}\right)$, the values of $\phi$ increase at $\mathrm{pH}$ values lower than the $\mathrm{pK}_{\mathrm{a}}$; for instance, at $\mathrm{pH}=6.5$ for $\Pi_{0}=20 \mathrm{mN} / \mathrm{m}$. Indeed, in the case of higher surface pressure, the compression increases the electrostatic potential at interface $\psi_{0}$, thereby decreasing the local proton concentration in the monolayer according to the Boltzmann law $\left[H^{+}\right]_{\text {interface }}=\left[H^{+}\right]_{\text {bulk }} \times \exp \left(-e \psi_{0} / k_{B} T\right)$, where $e=$ $1.6 \times 10^{-19} \mathrm{C}$ and $\left[\mathrm{H}^{+}\right]_{\text {bulk }}$ is the proton concentration far from the interface. In that case, the contribution of the electrostatic surface pressure is not negligible, and it follows that the $\mathrm{pK}_{\mathrm{a}}$ relative to the ionization of the cationic lipid is shifted to an apparent value $\left(\mathrm{pK}_{\mathrm{a}}^{\mathrm{app}}\right)$ due to the presence of a non-negligible surface electrostatic potential $\psi_{0}$, as

$\mathrm{pK}_{\mathrm{a}}^{\mathrm{app} .}=\mathrm{pK}_{\mathrm{a}}-\frac{e \psi_{0}}{2.30 k_{B} T}$

The shifts of the curves D and E (in Fig. 6b) occur as a consequence of the neutralization of the lipids to diminish the electrostatic potential induced by the drastic compression. This phenomenon actually follows the le Chatelier principle, whereby the system evolves as a function of the imposed external changes. In addition, it is interesting to note that $\psi_{0}$ is impacted by all the electrical charges present in the environment. In order to get qualitative information concerning how experimental parameters impact on the ionization of lipids (such 
as the counter-ion concentration and the lipid interfacial concentration), we used simple model (flat-plate model), detailed in the Supplementary information section. These calculations confirmed that, for more compressed layers, cationic lipids are neutralized at lower $\mathrm{pH}$.

Determination of specific area values and comparison with anionic lipid monolayers

These experiments were focused on the comparison of cationic lipid $\mathbf{1}$ and anionic lipid $\mathbf{2}$. Furthermore, in order to access the actual values of specific area $\omega$ without an influence of electrolyte, these experiments were performed without $\mathrm{NaCl}(\mathrm{pH}$ measurement was thus modified as follows: after the monolayer stabilization, $3 \mathrm{~mL}$ of bulk phase was collected in the external beaker and replaced by $3 \mathrm{~mL}$ of ultrapure water; the collected volume was mixed with $\mathrm{NaCl}$ solution to reach $50 \mathrm{mM}$ and the $\mathrm{pH}$ of the aliquot was measured). In the case of anionic lipid 2, the ionized homogeneous state corresponds to alkaline solutions, so the $\mathrm{pH}$ was adjusted to around $\mathrm{pH} 10$ to perform the initial compression isotherm, and then the protocol for lipid 1 was followed as the bulk phase $\mathrm{pH}$ was gradually decreased by adding given amounts of $\mathrm{HCl}$ to neutralize lipid 2.

A quantitative determination of the area per molecule required a suitable model. Reports in literature have shown that liquid-expanded monolayers (as in our case before phase transition) are satisfactorily described by the two-dimensional Volmer equation of state: ${ }^{15,26-31}$

$\Pi=\frac{k_{B} T}{A_{B} / N-\omega}-\Pi^{*}$

where $A_{B}$ is the bubble area, $N$ is the number of lipids present in the monolayer, and $\Pi^{*}$ is a constant called cohesion pressure that accounts for intermolecular interactions in gaseous or liquid-expanded states. In this context, an experimental set of $\Pi-A_{\mathrm{B}}$ curves as a function of $\mathrm{pH}$ were generated for both lipids in electrolyte-free bulk phase, as reported in the Supplementary information section (Fig. S2 and S3). The $\Pi-A_{\mathrm{B}}$ compression curves (at $\mathrm{pH}=3.07$ for lipid 1 and 10.06 for lipid 2) were then fitted with the Volmer equation Eq. (3) in the pressure range $0-10 \mathrm{mN} / \mathrm{m}$ (to ensure the applicability of Volmer's equation). The results are reported in Fig. $7 \mathrm{a}_{1}$ and Fig. $7 \mathrm{~b}_{1}$, respectively. The results give $N=5.27 \times 10^{13}$ molecules for lipid 1 shown in (a) and $N=3.02 \times 10^{13}$ molecules for lipid 2 in (b), which are in the same order of magnitude as reported in previously published experiments ${ }^{15}$ using exactly the same experimental setup with phospholipids and cholesterol. 
$\left(a_{1}\right)$

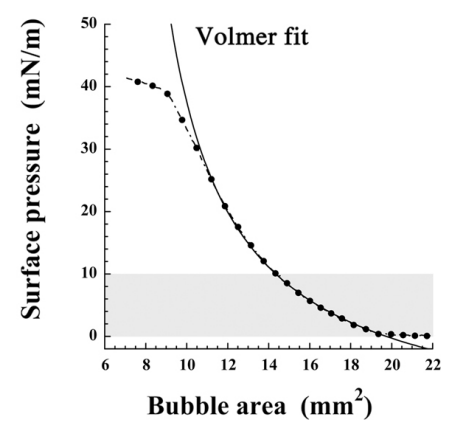

$\left(a_{2}\right)$

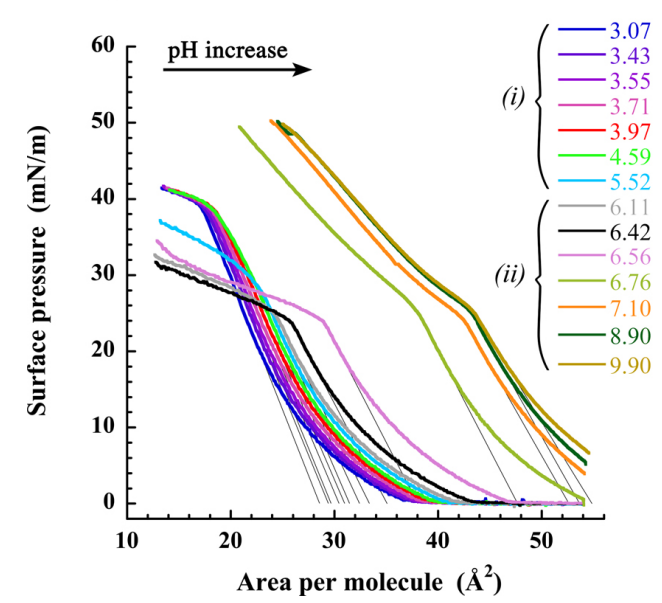

$\left(b_{1}\right)$

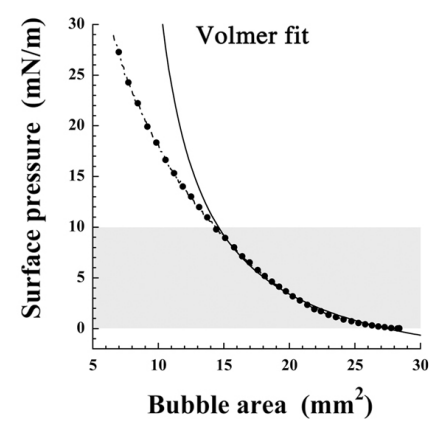

$\left(b_{2}\right)$

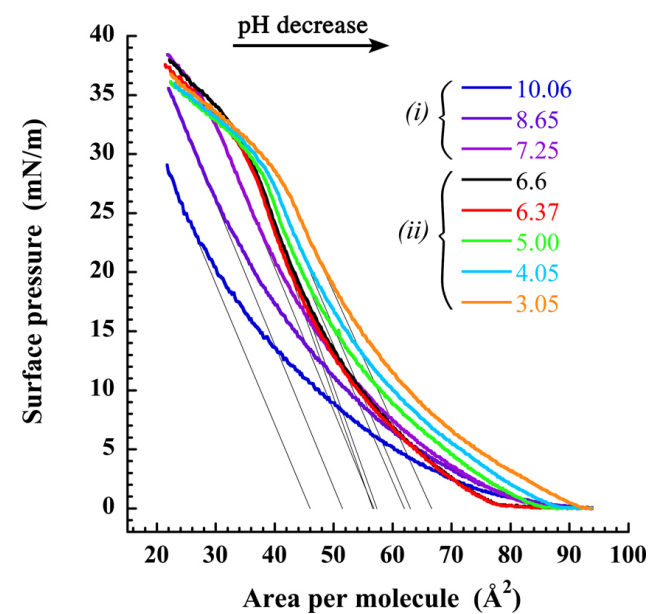

Figure 7: Study of (a) cationic lipid $\mathbf{1}$ and (b) anionic lipid $\mathbf{2}$ at the air / water interface without $\mathrm{NaCl}$ in the bulk phase, in function of $\mathrm{pH}$. ( $\left.\mathbf{a}_{1}\right)$ and $\left(\mathbf{b}_{1}\right)$ shows the fit with the Volmer's two-dimensional equation of state (Eq. 2) of the $\Pi-A_{\mathrm{B}}$ for fully ionized lipid, i.e., at $\mathrm{pH}=3.1$ for lipid $\mathbf{1}$ in $\left(\mathrm{a}_{1}\right)$, and at $\mathrm{pH}=10.1$ for lipid $\mathbf{2}$ in $\left(\mathrm{b}_{1}\right)$; the gray part shows the pressure range $(0-10 \mathrm{mN} / \mathrm{m})$ in which the Volmer equation is applied. $\left(\mathbf{a}_{2}\right)$ and $\left(\mathbf{b}_{2}\right)$ shows the $\Pi$ $A$ curves deduced from the values of $N$ obtained from the Volmer fit of $\left(\mathrm{a}_{1}\right)$ and $\left(\mathrm{b}_{1}\right)$.

Knowledge of $N$ for both lipids allows plotting of the corresponding $\Pi-A$ curves reported in Fig. $7 \mathrm{a}_{2}$ and Fig. $7 \mathrm{~b}_{2}$, with $A$ being the area per lipid. The $\Pi-A$ curve in Fig. $7 \mathrm{a}_{2}$ with cationic lipid 1 shows that the behavior is similar to that shown in Fig. 3 a with $50 \mathrm{mM} \mathrm{NaCl}$ in the bulk phase. For $\mathrm{pH}$ below 6.1, at a surface pressure lower than $40 \mathrm{mN} / \mathrm{m}$, the curve profiles are those of a homogeneous phase. They are gradually shifted to higher areas as the $\mathrm{pH}$ rises, and for $\mathrm{pH}$ values higher than $\mathrm{pH} 6.11$, a phase transition appears above a constant surface $\Pi \approx 25 \mathrm{mN} / \mathrm{m}$.

A notable difference from Fig. 3a is the presence of another phase transition, even for the fully ionized monolayers, at the higher compression range (for $\Pi \approx 40 \mathrm{mN} / \mathrm{m}$ ). Gathering our discussion above for higher compression, this phase transition can be a consequence of the high electrostatic potential induced by the compression itself that results in the neutralization of lipids. This behavior can be a consequence of the monolayer collapse (inducing, e.g., multilayer formation). As well, the rate at which the curves are shifted in response to the $\mathrm{pH}$ rise varies slightly from the previous results, as a small variation in rate is seen up to $\mathrm{pH}=6.42$, followed by a sudden shift above that $\mathrm{pH}$. The $\mathrm{pK}_{\mathrm{a}}^{\mathrm{app}}$. is then determined at the maximum variation rate (discussed below). 
In absence of electrolyte, it is shown that $\mathrm{pK}_{\mathrm{a}}^{\mathrm{app}} \approx 6.6$. By contrast, in presence of $\mathrm{NaCl}$ in the bulk phase, the $\mathrm{pK}_{\mathrm{a}}^{\mathrm{app} .} \approx$ 8.5. Considering Eq. (2), it follows that, in the absence of electrolyte, the electrostatic potential of the monolayer $\psi_{0}$ should be higher than it is in the presence of salts. This known behavior is understandable considering the charge screening undergone by the counterions on the charged cationic lipids. The impact on the global lipid molecules is a slight loss in their hydrophobicity (a salting-out effect), ${ }^{32}$ and a displacement toward the interface, which increases their excluded interfacial area similarly to the behavior observed above after neutralization. Such decrease of $\mathrm{pK}_{\mathrm{a}}^{\text {app }}$ for the lower $\mathrm{NaCl}$ concentrations is also predicted by the flat-plate model, detailed in the Supplementary information section.

Regarding the anionic lipid 2 , the $\mathrm{pK}_{\mathrm{a}}$ of its phenol group is expected to be about 10 , and its negative charge is neutralized by decreasing the $\mathrm{pH}$. The initial curve at $\mathrm{pH}=10.06$ confirms the expected homogeneous phase. Similarly to cationic lipid 1, the compression curves for lipid $\mathbf{2}$ are gradually shifted toward higher molecular areas as the $\mathrm{pH}$ decreases (i.e., along with the neutralization of the lipid charge). A similar behavior to that observed with the cationic lipid is evident; the interfacial molecular area per molecule gradually increases, with a possible explanation being a modification of the lipid conformation. An important difference exists, however, between the two lipids: (i) the curve shift vs. pH appears more monotonous than sigmoidal, complicating the determination of $\mathrm{pK}_{\mathrm{a}}^{\mathrm{app}}$. This different behavior can be interpreted by considering the chemical structure of the lipids (see Fig. 1). A short ethylene oxide chain composes a part of the polar head of lipid $\mathbf{2}$ (this is absent from lipid 1), thereby decreasing the impact of the $\mathrm{pH}$ on the lipid neutralization and the changes in the global hydrophilicity of lipid 2. It is interesting to note that surfactants with poly(ethylene oxide) chains can have two orientation states at the interface, depending on the surface pressure, that may potentially induce a transition between two states upon variations in the interfacial molecular area. ${ }^{33,34}$

At the higher surface pressures, a significant interval arises between experimental curve and Volmer's surface pressure predictions. As we have shown in a previous report, ${ }^{15}$ these results seem to indicate that $\omega$ is also a function of the surface pressure itself. This signifies that those lipids should present an ability for compression when the surface pressure is sufficiently high, in agreement with the expression ${ }^{35-37}$ linking $\omega$ and $\Pi$

$\omega=\omega_{0}(1-\epsilon \Pi)$

where $\omega_{0}$ is the value of specific area when $\Pi \rightarrow 0$, and $\epsilon$ is a compressibility factor. Combining Eq. (3) and (4) gives rise to $A=\frac{k_{B} T}{\Pi+\Pi^{*}}+\omega_{0}(1-\epsilon \Pi)$, where $A=A_{B} / N$, the area per molecule, which, for high surface pressure values, can be expressed as its linear form

$A=\omega_{0}(1-\epsilon \Pi)$

Determination of the excluded area per molecules $\omega_{0}$ is then possible from the $\Pi-A$ compression curves as the intersection between the tangent of the curve before the LE/LC transition (light gray lines in Fig. $7 a_{2}$ and $7 b_{2}$ ) and the horizontal line for $\Pi=0$. These values, determined in Fig. 7, are reported as the blue curves shown in Fig. 8. 


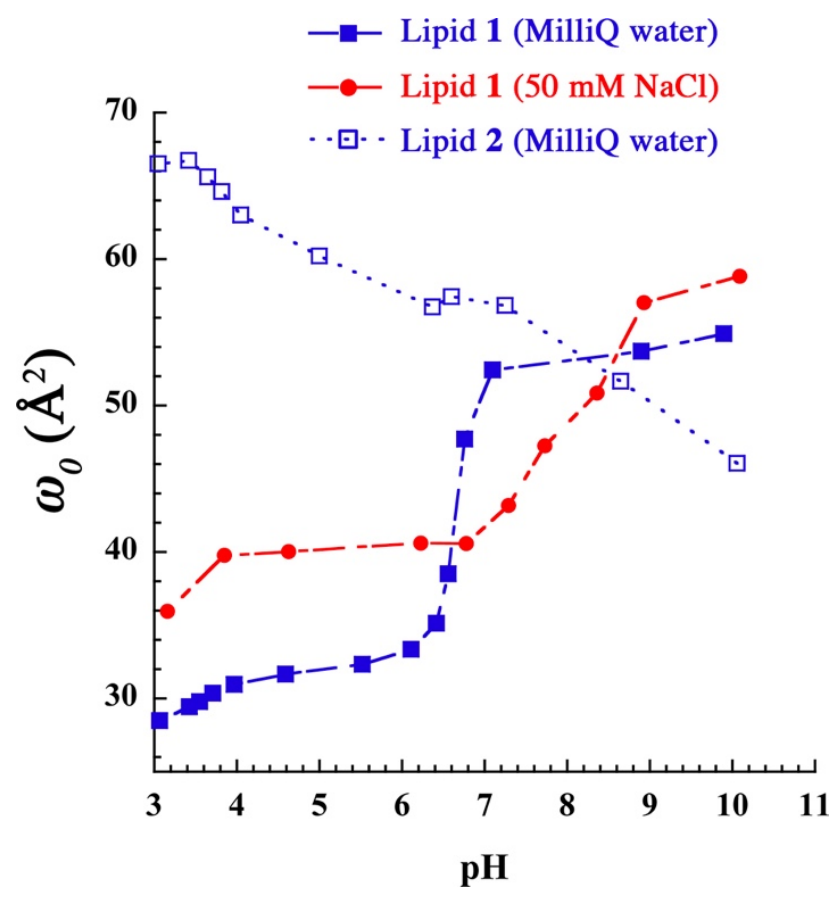

Figure 8: Excluded area per molecule $\omega_{0}$ against $\mathrm{pH}$, obtained for the fits of the compression curves (Fig. 7) and intersections of the gray lines with the abscissa $\Pi=0$. Blue curves are obtained from NaCl-free bulk phase (Fig. 7) and the red curve is obtained using a similar approach with $50 \mathrm{mN} \mathrm{NaCl}$ in the bulk phase (from Fig. 3).

Fig. 8 plots the experimental values of $\omega_{0}$ as a function of $\mathrm{pH}$ for both lipids. This representation confirms the previous observations. Lipid 1 presents variable specific areas $\omega_{0}$ depending on the ionization state. We observe a jump from around $\omega_{0} \approx 30 \AA^{2}$ for the lower $\mathrm{pH}$, to $\omega_{0} \approx 55 \AA^{2}$, following a sigmoidal transition. From this profile, we can read the apparent $\mathrm{pK}_{\mathrm{a}}$ value, $\mathrm{pK}_{\mathrm{a}}^{\mathrm{app} .} \approx 6.6$.

The anionic lipid 2 (open blue squares) shows the first increases of $\omega_{0}$ from $\sim 46$ to $\sim 57 \AA^{2}$ at $10>\mathrm{pH}>7$ (phase (i)), which then stabilize around $\sim 58 \pm 2 \AA^{2}$ for $7>\mathrm{pH}>5$, and finally increases again at $\mathrm{pH}<4$, to stabilize around $66 \AA^{2}$ for $\mathrm{pH}<3.5$. The increase in $\omega_{0}$ with decreasing $\mathrm{pH}$ is likely attributable to the loss in the bulk phase affinity due to the loss of electrostatic charge, thereby resulting in an increase in molecule confinement at interface and, thus, corroborating the idea of conformational change of the molecules. In addition, comparing the extent of lipid expansion due to $\mathrm{pH}$, the excluded area of lipid 2 increases to around $+26 \%$ $\left(\sim 46\right.$ to $\left.58 \AA^{2}\right)$, while it is much higher for lipid 1 , at $+83 \%$. As discussed above, this might be related to the presence of a short poly(ethylene oxide) chain in the polar head of lipid $\mathbf{2}$, which, to a certain extent, moderates the interfacial confinement effect during the neutralization process.

As a last remark, the comparison of the curve for lipid $\mathbf{1}$ with and without electrolyte in the bulk phase reveals a clear difference that corroborates the earlier results. (i) For the shift in the $\omega_{0}$ values at low pH: as noted in Fig. 3a, the addition of electrolyte shifts the compression profile to higher bubble areas. It follows that $\mathrm{NaCl}$ induces an increase in the area per molecule, as confirmed in Fig. 8, with a difference in the values of $\omega_{0}$. (ii) For the shift of two units in $\mathrm{pK}_{\mathrm{a}}^{\mathrm{app}}$. caused by the presence of electrolyte: this result corroborates our discussion above, and corroborate our hypothesis regarding the screening effect of salt on the monolayer and the 
induction of a change in the lipid location and orientation at the interface. This difference in $\mathrm{pK}_{\mathrm{a}}^{\mathrm{app}}$. shows that, when lipids are trapped in the interfacial region, their behavior can drastically change with the environmental conditions. Huge interfacial forces are applied to the molecules, able to impact on their interfacial orientation, up to influence their own properties like the value of $\mathrm{pK}_{\mathrm{a}}^{\mathrm{app}}$. Then, by contrast, for highest $\mathrm{pH}$, the two curves tend to merge, indicating that $\mathrm{NaCl}$ addition has a lower effect on hydrophilicity of the neutralized lipid $\mathbf{1}$. This is an expected behavior, since the electrolyte concentration has less effect on the hydrophobicity of a nonionic surfactant than of an ionic one.

\section{Conclusion}

To conclude, this work proposes an original and simple approach for studying the behavior of a monolayer - in our case, a monolayer of $\mathrm{pH}$-sensitive lipids - undergoing gradual changes in its environment. The original experimental procedure developed here relies on the deposition of insoluble lipids onto the water/air interface of a bubble maintained at a constant volume, followed by analysis with an axisymmetric drop shape analysis (ADSA) apparatus. The lipids studied displayed a $\mathrm{pH}$-sensitive functional group at the polar head. We propose several complementary approaches for studying the monolayer compression and dilatational elasticity at different $\mathrm{pH}$ values, and the effect of the presence of electrolyte in the aqueous bulk phase. The main results were related to the effect of lipid neutralization, which induces an increase in the excluded surface area per molecule possibly due to a modification of their location and orientation at the interface, as opposed to the decrease expected for charged lipids. This result is corroborated with all the different experimental approaches carried out, and allows determination of the values of $\mathrm{pK}_{\mathrm{a}}^{\mathrm{app}}$. and apparent $\mathrm{pK}_{\mathrm{a}}$ at the interface.

\section{Supporting information}

Calculation of the impact of the counter-ions concentration on the ionization of lipids, calculated with the flat-plate model. Isothermal compression curves, without $\mathrm{NaCl}$, for lipids $\mathbf{1}$ and $\mathbf{2}$, showing surface pressure vs. bubble area.

\section{Acknowledgement}

The authors would like to acknowledge the financial support provided by King Abdulaziz City for Science and Technology (KACST), Grant No. 14-MED1472-10.

\section{References}

(1) Longley, D. B.; Johnston, P. G. Molecular Mechanisms of Drug Resistance. J. Pathol. 2005, 205 (2), 275-292.

(2) Warburg, O. On the Origin of Cancer Cells. Science (80-. ). 1956, 123 (3191), 309314.

(3) Walsh, M.; Fais, S.; Spugnini, E. P.; Harguindey, S.; Abu Izneid, T.; Scacco, L.; Williams, P.; Allegrucci, C.; Rauch, C.; Omran, Z. Proton Pump Inhibitors for the Treatment of Cancer in Companion Animals. J. Exp. Clin. Cancer Res. 2015, 34, 93. 
(4) Omran, Z.; Scaife, P.; Stewart, S.; Rauch, C. Physical and Biological Characteristics of Multi Drug Resistance (MDR): An Integral Approach Considering PH and Drug Resistance in Cancer. Semin. Cancer Biol. 2017, 43, 42-48.

(5) Omran, Z.; Rauch, C. Acid-Mediated Lipinski's Second Rule: Application to Drug Design and Targeting in Cancer. Eur. Biophys. J. Biophys. Lett. 2014, 43 (4-5), 199206.

(6) Gerasimov, O. V; Rui, Y.; Thompson, D. H. Triggered Release from Liposomes Mediated by Physically and Chemically Induced Phase Transitions. In Vesicles; Rosoff, M., Ed.; Marcel Dekker: New York, 1996; pp 679-746.

(7) Shi, G.; Guo, W.; Stephenson, S. M.; Lee, R. J. Efficient Intracellular Drug and Gene Delivery Using Folate Receptor-Targeted PH-Sensitive Liposomes Composed of Cationic/Anionic Lipid Combinations. J. Control. Release 2002, 80, 309-319.

(8) Bi, H.; Ma, S.; Li, Q.; Han, X. Magnetically Triggered Drug Release from Biocompatible Microcapsules for Potential Cancer Therapeutics. J. Mater. Chem. B 2016, 4 (3269-3277).

(9) Bi, H.; Han, X. Magnetic Field Triggered Drug Release from Lipid Microcapsule Containing Lipid-Coated Magnetic Nanoparticles. Chem. Phys. Lett. 2018, 706, 455460.

(10) Simoes, S.; Moreira, J. N.; Fonseca, C.; Duzgunes, N.; de Lima, M. C. On the Formulation of PH-Sensitive Liposomes with Long Circulation Times. Adv. Drug Deliv. Rev. 2004, 56, 947-965.

(11) Rehman, A. U.; Omran, Z.; Anton, H.; Mély, Y.; Akram, S.; Vandamme, T. F.; Anton, N. Development of Doxorubicin Hydrochloride Loaded PH-Sensitive Liposomes: Investigation on the Impact of Chemical Nature of Lipids and Liposome Composition on PH-Sensitivity. Eur. J. Pharm. Biopharm. 2018, 133, 331-338. https://doi.org/https://doi.org/10.1016/j.ejpb.2018.11.001.

(12) Karanth, H.; Murthy, R. S. R. PH-Sensitive Liposomes-Principle and Application in Cancer Therapy. J. Pharm. Pharmacol. 2007, 59, 469-483.

(13) Koudelka, S.; Turanek Knotigova, P.; Masek, J.; Prochazka, L.; Lukac, R.; Miller, A. D.; Neuzil, J.; Turanek, J. Liposomal Delivery Systems for Anti-Cancer Analogues of Vitamin E. J. Control. Release 2015, 207, 59-69.

(14) de Oliveira Silva, J. C.; Miranda, S. E. M.; Leite, E. A.; de Paula Sabino, A.; Borges, K. B. G.; Cardoso, V. N.; Cassali, G. D.; Guimarães, A. G.; Oliveira, M. C.; de Barros, A. L. B. Toxicological Study of a New Doxorubicin-Loaded PH-Sensitive Liposome: A Preclinical Approach. Toxicol. Appl. Pharmacol. 2018, 352, 162-169.

(15) Anton, N.; Pierrat, P.; Lebeau, L.; Vandamme, T. F.; Bouriat, P. A Study of Insoluble Monolayers by Deposition at a Bubble Interface. Soft Matter 2013, 9 (42). https://doi.org/10.1039/c3sm51688a.

(16) Pierrat, P.; Lebeau, L. Characterization of Titratable Amphiphiles in Lipid Membranes by Fluorescence Spectroscopy. Langmuir 2015, 31 (45), 12362-12371. https://doi.org/10.1021/acs.langmuir.5b03258.

(17) Bouriat, P.; El Kerri, N.; Graciaa, A.; Lachaise, J. Properties of a Two-Dimensional Asphaltene Network at the Water-Cyclohexane Interface Deduced from Dynamic Tensiometry. Langmuir 2004, 20 (18), 7459-7464. https://doi.org/10.1021/la049017b.

(18) Defay, R.; Prigogine, I. Tension Superficielle et Adsorption; Editions Desoer: Liège, 1951.

(19) Levental, I.; Janmey, P. A.; Cēbers, A. Electrostatic Contribution to the Surface Pressure of Charged Monolayers Containing Polyphosphoinositides. Biophys. J. 2008, 
95 (3), 1199-1205. https://doi.org/https://doi.org/10.1529/biophysj.107.126615.

(20) Esker, A. R.; Zhang, L.-H.; Olsen, C. E.; No, K.; Yu, H. Static and Dynamic Properties of Calixarene Monolayers at the Air/Water Interface. 1. PH Effects with pDioctadecanoylcalix[4]Arene. Langmuir 1999, 15 (5), 1716-1724. https://doi.org/10.1021/la970016b.

(21) Kuroda, O.; Seto, H.; Narita, T.; Yamanaka, M.; Oishi, Y. Liposome Deformation by Imbalance of PH and Ionic Strength Across the Membrane. In Trends in Colloid and Interface Science XXIV; Starov, V., Procházka, K., Eds.; Springer Berlin Heidelberg: Berlin, Heidelberg, 2011; pp 49-53.

(22) Zimmermann, R.; Küttner, D.; Renner, L.; Kaufmann, M.; Zitzmann, J.; Müller, M.; Werner, C. Charging and Structure of Zwitterionic Supported Bilayer Lipid Membranes Studied by Streaming Current Measurements, Fluorescence Microscopy, and Attenuated Total Reflection Fourier Transform Infrared Spectroscopy. Biointerphases 2009, 4 (1), 1-6. https://doi.org/10.1116/1.3082042.

(23) Schweighofer, K. J.; Essmann, U.; Berkowitz, M. Simulation of Sodium Dodecyl Sulfate at the Water-Vapor and Water-Carbon Tetrachloride Interfaces at Low Surface Coverage. J. Phys. Chem. B 1997, 101 (19), 3793-3799. https://doi.org/10.1021/jp963460g.

(24) Davies, J. T.; Rideal, E. K. Interfacial Phenomena, Academic P.; New York, 1963.

(25) Liggieri, L.; Ravera, F.; Ferrari, M. Surface Rheology Investigation of the 2-D Phase Transition in n-Dodecanol Monolayers at the Water-Air Interface. Langmuir 2003, 19 (24), 10233-10240. https://doi.org/10.1021/la035442d.

(26) Fainerman, V. B.; Vollhardt, D. Equations of State for Langmuir Monolayers with Two-Dimensional Phase Transitions. J. Phys. Chem. B 1999, 103 (1), 145-150. https://doi.org/10.1021/jp983109q.

(27) Fainerman, V. B.; Vollhardt, D.; Melzer, V. Equation of State for Insoluble Monolayers of Aggregating Amphiphilic Molecules. J. Phys. Chem. 1996, 100 (38), 15478-15482. https://doi.org/10.1021/jp960523m.

(28) Gurkov, T. D.; Russev, S. C.; Danov, K. D.; Ivanov, I. B.; Campbell, B. Monolayers of Globular Proteins on the Air/Water Interface: Applicability of the Volmer Equation of State. Langmuir 2003, 19 (18), 7362-7369. https://doi.org/10.1021/la034250f.

(29) Guntupalli, R.; Sorokulova, I.; Long, R.; Olsen, E.; Neely, W.; Vodyanoy, V. Phage Langmuir Monolayers and Langmuir-Blodgett Films. Colloids Surf. B. Biointerfaces 2011, 82 (1), 182-189. https://doi.org/10.1016/j.colsurfb.2010.08.032.

(30) Valkova, L. A.; Zyablov, S. V; Erokhin, V. V; Koifman, O. I. Nanoaggregates in Floating Layers of Azaporphyrins. J. Porphyr. Phthalocyanines 2010, 14 (06), 513522. https://doi.org/10.1142/S1088424610002380.

(31) Valkova, L. A.; Glibin, A. S.; Koifman, O. I. Influence of the Solvent Nature on the Structure of Two-Dimensional Nanoaggregates in Langmuir Layers of Copper TetraTert-Butyltetrabenzotriazaporphyrin. Macroheterocycles 2011, 4 (3), 222-226.

(32) Salager, J. L. Quantifying the Concept of Physico-Chemical Formulation in SurfactantOil-Water Systems --- State of the Art. In Trends in Colloid and Interface Science X; Solans, C., Infante, M. R., García-Celma, M. J., Eds.; Steinkopff: Darmstadt, 1996; pp 137-142. https://doi.org/10.1007/BFb0115768.

(33) Miller, R.; Aksenenko, E. V; Liggieri, L.; Ravera, F.; Ferrari, M.; Fainerman, V. B. Effect of the Reorientation of Oxyethylated Alcohol Molecules within the Surface Layer on Equilibrium and Dynamic Surface Pressure. Langmuir 1999, 15 (4), 13281336. https://doi.org/10.1021/la980956b. 
(34) Fainerman, V. B.; Zholob, S. A.; Lucassen-Reynders, E. H.; Miller, R. Comparison of Various Models Describing the Adsorption of Surfactant Molecules Capable of Interfacial Reorientation. J. Colloid Interface Sci. 2003, 261 (1), 180-183. https://doi.org/https://doi.org/10.1016/S0021-9797(02)00226-6.

(35) Fainerman, V. B.; Miller, R.; Kovalchuk, V. I. Influence of the Compressibility of Adsorbed Layers on the Surface Dilational Elasticity. Langmuir 2002, 18 (20), 77487752. https://doi.org/10.1021/la020024e.

(36) Fainerman, V. B.; Miller, R.; Kovalchuk, V. I. Influence of the Two-Dimensional Compressibility on the Surface Pressure Isotherm and Dilational Elasticity of Dodecyldimethylphosphine Oxide. J. Phys. Chem. B 2003, 107 (25), 6119-6121. https://doi.org/10.1021/jp021876q.

(37) Pradines, V.; Krägel, J.; Fainerman, V. B.; Miller, R. Interfacial Properties of Mixed $\beta$ Lactoglobulin-SDS Layers at the Water/Air and Water/Oil Interface. J. Phys. Chem. $B$ 2009, 113 (3), 745-751. https://doi.org/10.1021/jp8091573. 